\title{
Comparison of magnetic resonance imaging and computed tomography in suspected lesions in the posterior cranial fossa
}

\author{
Graham M Teasdale, Donald M Hadley, Audrey Lawrence, Ian Bone, Helen Burton, \\ Robin Grant, Barrie Condon, Peter Macpherson, Jack Rowan
}

\begin{abstract}
Objective-To compare computed tomography and magnetic resonance imaging in investigating patients suspected of having a lesion in the posterior cranial fossa.

Design-Randomised allocation of newly referred patients to undergo either computed tomography or magnetic resonance imaging; the alternative investigation was performed subsequently only in response to a request from the referring doctor.

Setting-A regional neuroscience centre serving $2 \cdot 7$ million.

Patients-1020 Patients recruited between April 1986 and December 1987, all suspected by neurologists, neurosurgeons, or other specialists of having a lesion in the posterior fossa and referred for neuroradiology. The groups allocated to undergo computed tomography or magnetic resonance imaging were well matched in distributions of age, sex, specialty of referring doctor, investigation as an inpatient or an outpatient, suspected site of lesion, and presumed disease process; the referring doctor's confidence in the initial clinical diagnosis was also similar.
\end{abstract}

Interventions-After the patients had been imaged by either computed tomography or magnetic resonance (using a resistive magnet of $0.15 \mathrm{~T}$ ) doctors were given the radiologist's report and a form asking if they considered that imaging with the alternative technique was necessary and, if so, why; it also asked for their current diagnoses and their confidence in them.

Main outcome measures - Number of requests for the alternative method of investigation. Assessment of characteristics of patients for whom further imaging was requested and lesions that were suspected initially and how the results of the second imaging affected clinicians' and radiologists' opinions.

Results-Ninety three of the 501 patients who initially underwent computed tomography were referred subsequently for magnetic resonance imaging whereas only 28 of the 493 patients who initially underwent magnetic resonance imaging were referred subsequently for computed tomography. Over the study the number of patients referred for magnetic resonance imaging after computed tomography increased but requests for computed tomography after magnetic resonance imaging decreased. The reason that clinicians gave most commonly for requesting further imaging by magnetic resonance was that the results of the initial computed tomography failed to exclude their suspected diagnosis (64 patients). This was less common in patients investigated initially by magnetic resonance imaging (eight patients). Management of 28 patients $(6 \%)$ imaged initially with computed tomography and 12 patients (2\%) imaged initially with magnetic resonance was changed on the basis of the results of the alternative imaging.

Conclusions-Magnetic resonance imaging provided doctors with the information required to manage patients suspected of having a lesion in the posterior fossa more commonly than computed tomography, but computed tomography alone was satisfactory in $80 \%$ of cases. Magnetic resonance imaging is a useful alternative to computed tomography and has advantages over it in patients suspected of having a lesion in the posterior cranial fossa.

\section{Introduction}

Magnetic resonance imaging portrays intracranial structures with striking clarity, but after eight years of clinical experience and numerous enthusiastic reports its appropriate use remains uncertain and controversial. ${ }^{12}$ Recent reviews and editorials have criticised the shortage of reliable information about its diagnostic performance, its clinical efficacy and efficiency, and, above all, its effects on management of patients. ${ }^{1-6}$ We report a prospective randomised study in which we compared magnetic resonance imaging and computed tomography in investigating patients referred to us who were suspected of having a lesion in the posterior cranial fossa, where magnetic resonance imaging is believed to be of particular value.

The main criterion that we used to compare magnetic resonance imaging and computed tomography was whether or not the information yielded by one investigation gave the referring clinicians sufficient confidence in their diagnoses to manage the patients without further cross sectional imaging. When additional imaging was requested we audited how the results influenced diagnosis and management.

\section{Methods}

Patients were enrolled between April 1986 and December 1987 at the institute, which is the regional centre providing inpatient and outpatient facilities for neurosurgical and neurological investigation and treatment for a population of 2.7 million in the west of Scotland. Magnetic resonance imaging was established in 1984 , and before this study 200 patients suspected of having a lesion in the posterior fossa had been examined with both this type of imaging and computed tomography. ${ }^{8}$ The aims and design of the study were agreed in advance by all of the participating consultants (seven neurosurgeons, 10 neurologists, three neurootologists, one neuro-ophthalmologist, and four neuroradiologists). It was approved by the institute's research ethics committee, and informed consent for the examination with magnetic resonance imaging was
Correspondence to:

Professor Teasdale.

Br.Med f 1989;299:349-55 
obtained from the patient or, if the patient was a child or confused, from the next of kin.

Patients with a suspected lesion in the posterior fossa referred to the department of neuroradiology for investigation were identified from a modified request card. On this clinicians recorded the clinical features, the likely location and pathological nature of any lesion, and their confidence in their provisional diagnosis. A member of the department's clerical staff used a randomisation list to allocate patients to either magnetic resonance imaging or computed tomography. After the first investigation the clinicians were provided with the radiologist's report and a form asking if they considered the alternative imaging necessary. When they requested the alternative technique they were required to state the reasons and their currently held views about the site of the lesion and its pathological nature and their confidence in the diagnosis. The radiologist conducting the second examination had access to the report of the first. After receiving the report on the second examination the clinicians answered further questions about how they planned to treat the patient. Based on the information available when the patient was discharged they filled in a final form, giving their views about the site and pathological nature of any lesion and their confidence in the final diagnosis.

Computed tomography was carried out with a Tomoscan 350 (Philips). Routinely 16 transaxial sections $6 \mathrm{~mm}$ thick were imaged from the foramen magnum to the vertex, parallel to a line from the external auditory meatus to the glabella (about $10^{\circ}$ from the radiographic baseline). When small lesions were suspected or the internal auditory meatuses were examined contiguous or overlapping sections $3 \mathrm{~mm}$ thick were used. Contrast enhancement was carried out at the radiologist's discretion with doses of iodine varying from $15 \mathrm{~g}$ to $31 \mathrm{~g}$; delayed postcontrast scanning was not done. Occasionally direct coronal and sagittal images were used as well as reformatted images.

Magnetic resonance imaging was carried out with a $0.15 \mathrm{~T}$ Vista 1100 resistive magnet (Picker) operating at $6.38 \mathrm{MHz}$. A two dimensional Fourier transform collecting mode with two repetitions was used, and the data were acquired on a 128 or $192 \times 256$ matrix interpolated to a $256 \times 256$ display with a field of view of

TABLE I-Clinical features and doctors' initial diagnoses and confidence in their diagnoses in 1020 patients suspected of having lesion in posterior fossa and allocated to undergo either computed tomography or magnetic resonance imaging. Figures are numbers (percentages) of patients

\begin{tabular}{lcc}
\hline & $\begin{array}{c}\text { Patients randomised to undergo } \\
\text { computed tomography } \\
(\mathbf{n}=511)\end{array}$ & $\begin{array}{c}\text { Patients randomised to undergo } \\
\text { magnetic resonance } \\
(\mathbf{n}=509)\end{array}$ \\
\hline Male & $235(46)$ & $229(45)$ \\
Referred from department of neurosurgery & $73(14)$ & $66(13)$ \\
Referred from department of neurology & $424(83)$ & $417(82)$ \\
Referred from other departments & $14(3)$ & $26(5)$ \\
Inpatients & $238(47)$ & $219(43)$ \\
Suspected site of lesion: & $211(41)$ & $243(48)$ \\
Brain stem & $117(23)$ & $113(22)$ \\
Cerebellum & $99(19)$ & $85(17)$ \\
Cerebellopontine angle/VIIIth nerve & $26(5)$ & $34(7)$ \\
Other cranial nerve & $46(9)$ & $30(6)$ \\
Craniocervical junction & $9(2)$ & $4(1)$ \\
NA & $126(25)$ & $153(30)$ \\
Suspected disease: & $108(21)$ & $96(19)$ \\
Vascular & $78(15)$ & $88(17)$ \\
Tumour & $50(10)$ & $48(9)$ \\
Demyelination & $42(8)$ & $32(6)$ \\
Degeneration & $85(16)$ & $77(15)$ \\
Congenital & $22(4)$ & $15(3)$ \\
Other & & \\
NA/unknown & $80(16)$ & $91(18)$ \\
Confidence in diagnosis: & $154(30)$ & $125(24)$ \\
$<40 \%$ & $170(33)$ & $174(34)$ \\
$40-60 \%$ & $99(19)$ & $108(21)$ \\
60-80\% & & \\
$>80 \%$ & &
\end{tabular}

$\mathrm{NA}=$ Not available.

$\mathrm{NA}=\mathrm{Not}$ available.
${ }^{\star}$ Includes infections and traumatic and toxic causes.
$30 \mathrm{~cm}$. Close fitting helmet ("Jedi”) receiving coils that were tailored to the patient's head size were used throughout. Routinely we obtained two sets of $8 \mathrm{~mm}$ thick axial images. The first set was a transverse magnetisation relaxation (T2) time weighted spin echo (SE2000/80) set containing 16 contiguous slices that covered the whole head from the vertex to the first cervical vertebra. The second set was a longitudinal magnetisation relaxation $(\mathrm{T} 1)$ time weighted inversion recovery (IR1660/400/40) set containing eight contiguous slices centred on the position of interest and that usually included the exits of the fourth ventricle and the body of the corpus callosum. In selected cases we used sets of high resolution images in orthogonal or oblique orientations, such as $5 \mathrm{~mm}$ thick multiple slice balanced spin echo (SE 700/32) sequences with a $256 \times 256$ matrix and a field of view of $25 \mathrm{~cm}$ with a $12 \mathrm{~cm}$ diameter single turn surface receiver coil, to image the intracanalicular auditory nerves and other sites. Contrast enhancement of the images with gadolinium diethylenetriaminepentaacetic acid was not available.

The study was designed on an intention to image basis. The aim was to find out, within a reasonable time, if there was a difference in the rate at which a second request for imaging was made, depending on whether the patient had been first allocated to computed tomography or magnetic resonance imaging. As the first 115 patients entered into the study indicated that the rate of requests for a second investigation would be between 15 and $20 \%$, the size of the sample was set at 1000 to detect a reduction in the rate of between $5 \%$ and $10 \%$ with a minimum power of $75 \%$. Confidence intervals are given for proportions, and comparisons were made with the $\chi^{2}$ test with analysis of the standardised residuals ${ }^{9}$; reported differences were significant at the $5 \%$ level.

Six months after entry to the study information about each patient's condition and diagnosis was sought from the general practitioners.

\section{Results}

PATIENTS

A total of 1020 patients were entered into the study; 511 (mean (SD) age $48 \cdot 4(15 \cdot 6)$ years) were randomised to undergo computed tomography and 509 (mean age $48.6(16.0)$ years) to undergo magnetic resonance imaging; these comprised $5 \%$ of patients undergoing cranial computed tomography and $30 \%$ of those undergoing magnetic resonance imaging during the same period. Most of the patients in both groups had been referred by neurologists, and roughly equal proportions were inpatients and outpatients (table I); the suspected sites of lesions, diagnoses, and doctors' confidence in their diagnoses were similar in both groups. Twenty three doctors referred patients for scanning. The number of requests per doctor ranged from one (two doctors) to 182 (median 25 , interquartile range 5-69). For individual doctors similar numbers of patients were randomised to computed tomography or magnetic resonance imaging. Forty eight patients with a suspected lesion in the posterior fossa attended the institute but did not enter the study: 22 of these had undergone computed tomography before referral; 21 had been admitted out of hours as emergencies when magnetic resonance imaging was not available; and five were eligible but were imaged by computed tomography in error without having been entered into the study.

Of the 511 patients randomised to undergo computed tomography, $501(98 \%)$ were examined by this technique; four underwent magnetic resonance imaging instead (three attended when the computed tomographic scanner was not working and one was 
pregnant), and six did not attend for scanning (figure). Of the 509 patients randomised to undergo magnetic resonance imaging, $493(97 \%)$ were examined by this technique: 11 had computed tomography instead (six had claustrophobia in the magnetic resonance imager; two attended when the imager was not working; one had an electronic implant; and two had computed tomography because of an administrative error), and five were not imaged (one died; one had a stroke; one refused both types of imaging; and two did not keep their apointments).

\section{RESULTS OF INITIAL IMAGING}

The images were normal in most patients in both groups (table II). More vascular and demyelinating diseases were diagnosed in patients imaged by magnetic resonance. The less common finding of cerebellar atrophy in patients imaged by magnetic resonance reflected one of the radiologists' more conservative attitude; a separate analysis showed that this observer also had a high threshold for diagnosing atrophy on computed tomography.

\section{REQUESTS FOR INVESTIGATION BY ALTERNATIVE TECHNIQUE}

Of the 501 patients who underwent computed tomography, $93(19 \%$; $95 \%$ confidence interval 15 to $22 \%$ ) were referred for magnetic resonance imaging and this was done in 90 cases. Of the 493 patients who underwent magnetic resonance imaging, $28(6 \% ; 3 \cdot 7$ to $7 \cdot 7 \%$ ) were referred for and underwent computed tomography. The difference between the proportions of requests for computed tomography after magnetic resonance imaging and for magnetic resonance imaging after computed tomography was $13 \%$ (9 to $17 \%$ ). This difference did not change even when the patients who were imaged by the technique other than that to which they had been allocated were regarded as "failures." The proportion of patients referred for magnetic resonance imaging after computed tomography generally increased with time, whereas the proportion who underwent computed tomography after magnetic resonance imaging decreased (table III).

Sixteen clinicians requested the alternative imaging technique; the seven others had initially referred only between one and 10 patients each. The number of second requests for imaging from each doctor ranged from one (three doctors) to 26 (median 6.5 ; interquartile range $3 \cdot 5-8 \cdot 5)$. Four neurologists had referred 550 of the patients and made 59 of the requests
TABLE II-Diagnoses made on basis of results of initial computed tomography or magnetic resonance imaging of patients suspected of having lesion in posterior fossa

\begin{tabular}{lcc}
\hline & $\begin{array}{c}\text { Imaged by } \\
\text { computed tomography } \\
(\mathrm{n}=501)\end{array}$ & $\begin{array}{c}\text { Imaged by } \\
\text { magnetic resonance } \\
(\mathrm{n}=493)\end{array}$ \\
\hline Normal & 332 & 316 \\
Atrophy & 109 & 56 \\
Vascular disease & 22 & 52 \\
Tumour & 22 & 28 \\
Demyelination & 2 & 24 \\
Congenital disease & 7 & 12 \\
Other & 7 & 5 \\
\hline
\end{tabular}

*Includes infection, trauma, and hydrocephalus.

TABLE III - Numbers of patients for whom alternative imaging was requested after computed tomography or magnetic resonance imaging in successive groups of 100 patients admitted to study

\begin{tabular}{ccc}
\hline & \multicolumn{2}{c}{ First examination } \\
\cline { 2 - 3 } $\begin{array}{c}\text { Successive } \\
\text { groups of } \\
\text { patients }\end{array}$ & $\begin{array}{c}\text { Computed tomography } \\
(\mathrm{n}=501)\end{array}$ & $\begin{array}{c}\text { Magnetic resonance imaging } \\
(\mathrm{n}=493)\end{array}$ \\
\hline-100 & 8 & 7 \\
-200 & 6 & 5 \\
-300 & 8 & 2 \\
-400 & 8 & 3 \\
-500 & 9 & 4 \\
-600 & 9 & 1 \\
-700 & 12 & 2 \\
-800 & 12 & 4 \\
-900 & 11 & \\
-1000 & 10 & \\
\hline
\end{tabular}

for the alternative imaging technique. The number of requests for the alternative imaging technique that each of these neurologists made for patients whom they had referred and who had had computed tomography initially was respectively 19/62, 10/51; $8 / 69$, and $6 / 93$, and for patients who had had magnetic resonance imaging initially it was $7 / 55,6 / 61,1 / 76$, and $2 / 83$. Each of the 12 other doctors who requested the alternative imaging technique also more often asked for magnetic resonance imaging than computed tomography. Table IV shows that the reason that clinicians gave most commonly for requesting magnetic resonance imaging after computed tomography was that computed tomography had not excluded their suspected diagnosis; this reason was given less commonly by those who requested computed tomography after magnetic resonance imaging. In both cases the doctor's confidence in the diagnosis had not been increased greatly by the results of the first

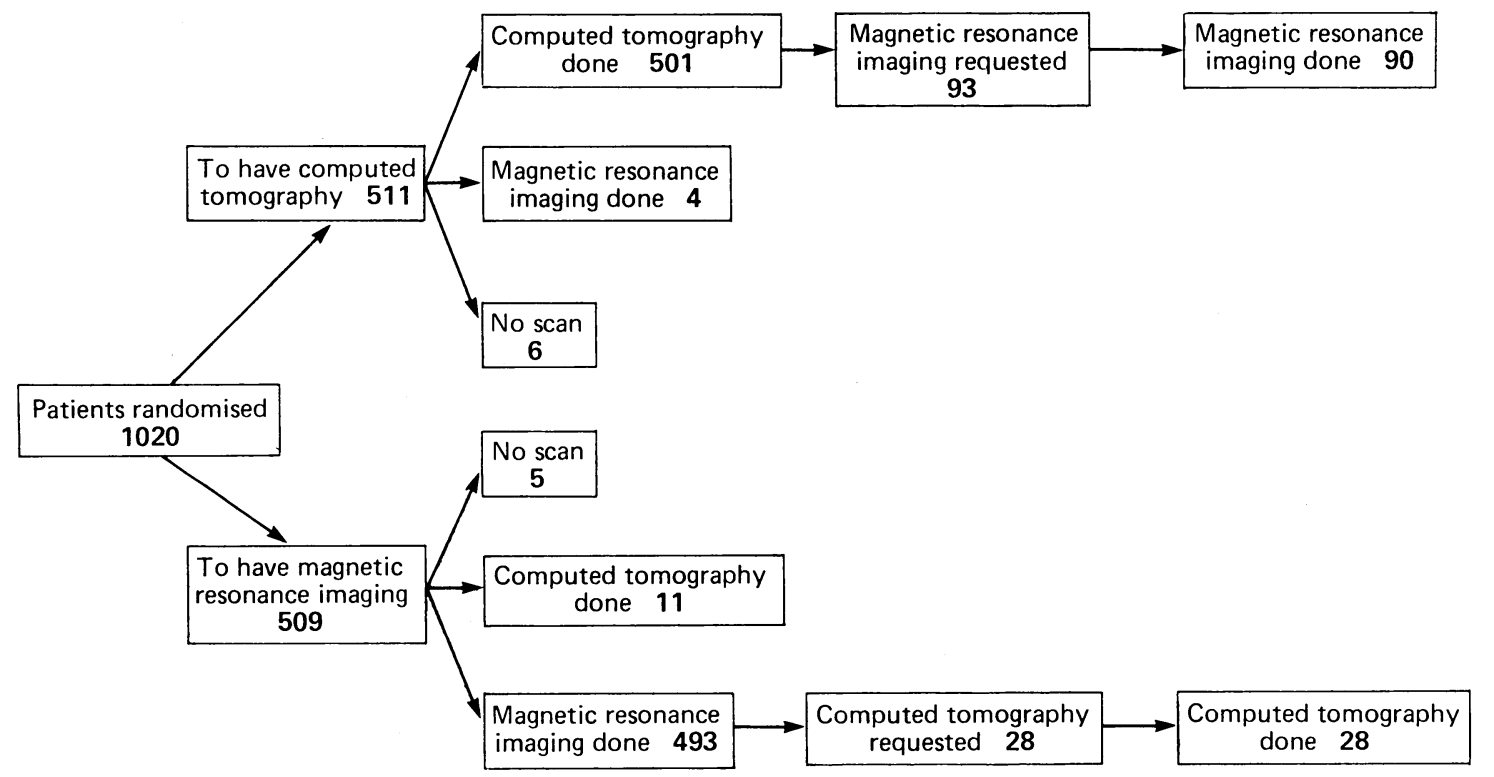

Investigations performed in patients randomised to have firstly either computed tomography or magnetic resonance imaging 
TABLE IY -Doctors' reasons for referring patients suspected of having lesion in posterior fossa for alternative imaging after they had undergone computed tomography or magnetic resonance imaging and doctors confidence in their diagnoses after initial imaging. Figures are numbers (jercentages) of patients

\begin{tabular}{|c|c|c|}
\hline & $\begin{array}{l}\text { Patients referred for } \\
\text { magnetic resonance imaging } \\
(\mathrm{n}=93)\end{array}$ & $\begin{array}{l}\text { Patients referred for } \\
\text { computed tomography } \\
n=28\end{array}$ \\
\hline \multicolumn{3}{|l|}{ Reason for referral: } \\
\hline Clinical diagnosis not excluded by first result & $6+69$ & 8.29: \\
\hline First result not sufficiently specific & $56 \cdot 60$ & $17: 61$ \\
\hline First result conflicted with clinical diagnosis & 2325 & 1036 \\
\hline \multicolumn{3}{|l|}{ Possible change of management: } \\
\hline Present plan medical & $39+2$ & $932)$ \\
\hline Present plan an operation & 3133 & 725 \\
\hline \multicolumn{3}{|l|}{ Confidence in diagnosis: } \\
\hline$<40 \%$ & $16(18)$ & $3(11$ \\
\hline $40-60 \%$ & 1819 & 725 \\
\hline $60-80 \%$ & 2830 & $6(21)$ \\
\hline$>80 \%$ & $30(32)$ & $10(36)$ \\
\hline $\mathrm{NA}$ & & 27 \\
\hline
\end{tabular}

$\mathrm{NA}=$ Not available
$67(20 \%)$ were referred for magnetic resonance imaging, but computed tomography was requested for only $13(4 \%)$ of the 316 patients whose initial magnetic resonance image was normal.

EFFECT OF RESULTS OF INVESTIGATION BY ALTERNATIVE IMAGING TECHNIQUE

The neuroradiologist's opinion changed in $21(23 \%)$ of the 90 patients who underwent magnetic resonance imaging after computed tomography, and this led to important changes in diagnosis. Thus magnetic resonance imaging identified one acoustic neuroma and one malignant tumour of the base of the skull which were not shown by computed tomography, excluded two tumours of the cerebellopontine angle that were suspected after computed tomography, and identified a congenital Arnold-Chiari malformation in two patients and meningoencephalitis in one. It also detected demyelinating lesions in seven patients and brain stem or cerebellar infarction in five patients. Magnetic resonance images were normal or showed only atrophy in $50(78 \%)$ of the 64 patients referred after these diagnoses had been made on computed tomography.

The diagnosis was changed in five $(18 \%)$ of the 28 patients who underwent computed tomography after magnetic resonance imaging. Even in these cases computed tomography was usually less informative: one patient shown by magnetic resonance imaging to have multiple metastases was thought on computed tomography to have multiple infarcts, but subsequently the diagnosis on magnetic resonance imaging was confirmed; one patient whose magnetic resonance image showed demyeliration had a normal computed tomogram; and one patient whose magnetic resonance images showed a small brain stem infarct had a normal computed tomogram.

Clinicians' assessments of the value of the second investigation showed broadly similar patterns in the two groups. The doctors changed their diagnoses in 42 of the 90 patients $(47 \%)$ who underwent magnetic resonance imaging after computed tomography and in nine of the $28(32 \%)$ who underwent computed tomography after magnetic resonance imaging. In 24 patients clinically suspected of having a tumour after computed tomography another diagnosis was established after magnetic resonance imaging in 15 , whereas computed tomography changed the diagnosis in only one of seven patients thought to have a tumour after magnetic resonance imaging. Twenty five patients diagnosed as having demyelination were referred after computed tomography for magnetic resonance imaging, which substantiated the diagnosis in 17; one of the remaining patients was shown to have carcinomatous meningitis and another meningoencephalitis.

The alternative imaging changed the management that the doctors had planned in a minority of patients (table V). Changes were reported for 28 of the 93 patients referred for magnetic resonance imaging after computed tomography $(6 \%$ of the 511 patients who were randomised to undergo computed tomography). Three patients' management was changed to a plan for an operation: one of them was shown by magnetic resonance imaging to have an acoustic neuroma that had not been shown by computed tomography; in another an Arnold-Chiari malformation and basilar invagination were shown more clearly by magnetic resonance imaging; and the other patient had an arachnoid cyst that was shown by both investigations. Management of 11 patients was changed to a conservative or medical approach: four of these had no abnormality on either investigation (apart from two who had atrophy); one was thought on computed tomography to have an acoustic neuroma but had 
normal magnetic resonance images; one, whose computed tomogram was normal, was shown by magnetic resonance imaging to have an Arnold-Chiari malformation without a syrinx; three had an intrinsic tumour in either the brain stem (two) or cerebellum (one); and one had a small en plaque tentorial tumour on both investigations.

The management plan was changed for 12 of the 28 patients referred for computed tomography after magnetic resonance imaging $(2 \%$ of the 509 patients randomised to this technique). The three patients for whom it was changed to an intention to operate had similar findings with both techniques: a brain stem tumour, a subarachnoid haemorrhage with a temporal haematoma, and a thalamic lesion. Of the three patients whose management was changed from an operation to medical or conservative management after computed tomography, one had a malignant cerebellar tumour shown by both techniques, one had a normal posterior fossa with both techniques, and one had atrophy on computed tomography whereas the magnetic resonance images were normal.

\section{OPERATIONS}

A major intracranial operation was performed on 17 patients who had been randomised to undergo computed tomography (five of whom also underwent magnetic resonance imaging) and on 12 of those randomised to undergo magnetic resonance imaging (two of whom also underwent computed tomography). Seven patients in each group had a minor neurosurgical operation, two after undergoing magnetic resonance

TABLE V-Doctors' plans for managing patients suspected of having lesion in posterior fossa before and after imaging by alternative technique to initial imaging

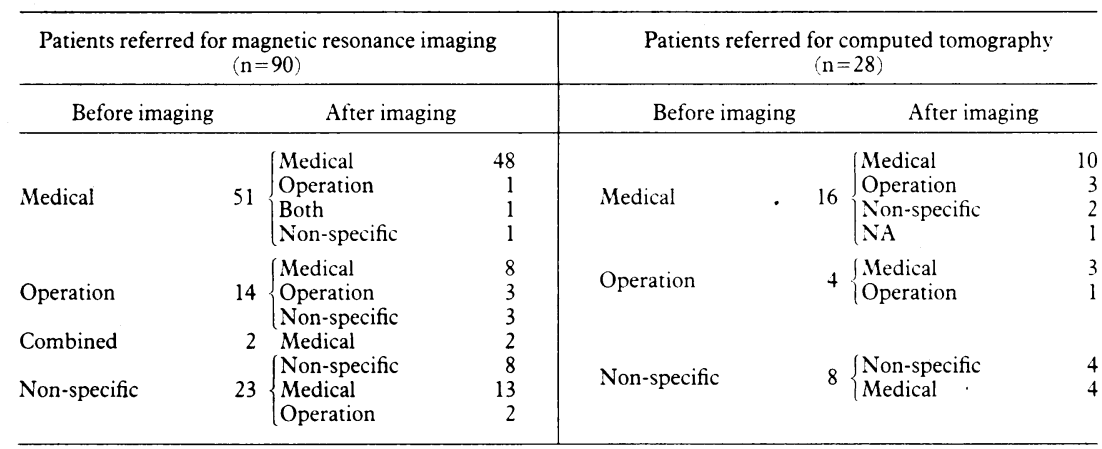

$\mathrm{NA}=$ Not available

TABLE VI-Final diagnoses and doctors' confidence in them in 1020 patients suspected of having lesion in posterior fossa and allocated to undergo either computed tomography or magnetic resonance imaging. Figures are numbers (percentages) of patients

\begin{tabular}{|c|c|c|}
\hline & $\begin{array}{l}\text { Patients randomised to undergo } \\
\text { computed tomography } \\
\qquad(\mathrm{n}=511)\end{array}$ & $\begin{array}{l}\text { Patients randomised to undergo } \\
\text { magnetic resonance imaging } \\
\qquad(\mathrm{n}=509)\end{array}$ \\
\hline \multicolumn{3}{|l|}{ Site of lesion: } \\
\hline Brain stem & $138(27)$ & $1+6(29)$ \\
\hline Cerebellum & $77(15)$ & $84(16)$ \\
\hline Cerebellopontine angle/VIIIth nerve & $29(6)$ & 20 \\
\hline Craniocervical junction & $9(2)$ & $5(1)$ \\
\hline Other & $112(22)$ & $122(24)$ \\
\hline Unknown & $112(22)$ & $101(20)$ \\
\hline NA & $34(7)$ & 316 \\
\hline \multicolumn{3}{|l|}{ Disease: } \\
\hline Vascular & $87(17)$ & $119(23)$ \\
\hline Tumour & $29(6)$ & $31(6)$ \\
\hline Demyelination & $34(7)$ & $46(9)$ \\
\hline Degeneration & $21(4)$ & $20(4)$ \\
\hline Other* & $165(32)$ & $146(29)$ \\
\hline Unknown & $160(31)$ & $132(26)$ \\
\hline $\mathrm{NA}$ & $15(3)$ & $15(3)$ \\
\hline \multicolumn{3}{|l|}{ Confidence in diagnosis: } \\
\hline$<40 \%$ & $42(8)$ & 428 \\
\hline $40-60 \%$ & $+2(8)$ & 479 \\
\hline $60-80 \%$ & $101(20)$ & $97(19)$ \\
\hline$>80 \%$ & $242(47)$ & $268(53)$ \\
\hline $\mathrm{NA}$ & $84(16)$ & $55(11)$ \\
\hline
\end{tabular}

$\mathrm{NA}=$ Not available

$\star$ Includes congenital infection, trauma, and toxic causes. imaging as the second examination and one after undergoing computed tomography as the second examination.

\section{DIAGNOSES AT DISCHARGE AND FOLLOW UP}

Table VI shows that the doctors' final diagnoses had broadly similar patterns of the site of the lesion, pathological process, and confidence in their diagnosis in both groups. Patients who were allocated to undergo computed tomography had a vascular disorder diagnosed less commonly than those allocated to undergo magnetic resonance imaging, and more were left without a diagnosis, though the difference was not significant. Indeed, the main difference between the initial (table I) and final diagnoses was that the proportion of cases in which the diagnosis was unknown increased 10-fold, mainly because suspected tumours or demyelination were excluded. Doctors were more than $80 \%$ confident of their diagnosis in half of the patients at discharge, which was twice the proportion at the time of the first investigation; the figure was similar whether only one or both investigations had been performed. The only notable changes in diagnosis reported at the six month follow up were from unknown to vascular disease in three patients, from vascular disease to demyelination in one patient, and from injury to infection in one.

\section{Discussion}

The results show that the doctors clearly preferred magnetic resonance imaging for investigating patients suspected of having a lesion in the posterior cranial fossa. Doctors were willing to manage patients on the basis of results of magnetic resonance imaging alone more often than on the basis of results of computed tomography alone, and magnetic resonance imaging was requested three times more commonly after computed tomography than computed tomography after magnetic resonance imaging. Diagnosis and management were changed in a few cases, but the changes were more substantial in patients imaged by magnetic resonance after computed tomography. Nevertheless, our results also show that computed tomography was considered satisfactory for $83 \%$ of the patients.

Calls for rigorous assessment of new technologies are common, ${ }^{10-12}$ but successful examples are rare. ${ }^{13}$ To our knowledge no other study has compared prospectively computed tomography and magnetic resonance imaging performed at random in investigating an unselected population of patients suspected of having an intracranial lesion. In many previous studies patients were selected, and often it is not clear if the contributions of computed tomography and magnetic resonance imaging were assessed separately and prospectively or if magnetic resonance imaging was performed and interpreted in the light of previous computed tomography. In an analysis of 80 separate assessments of magnetic resonance imaging ${ }^{1}$ all but six were found to contain one or more of three main methodological errors ${ }^{14}$ : "work up" bias, "diagnostic review" bias, and "test review" bias. Similarly, when 10 commonly accepted criteria for research methods were applied to 54 studies of magnetic resonance imaging none of the studies satisfied more than five of them and $90 \%$ of the reports were deficient in eight or more. ${ }^{+}$

Three studies have compared the diagnostic performances of computed tomography and magnetic resonance imaging in patients with a range of intracranial disorders, including lesions in the posterior fossa, but in each study the source of the patients is unclear and each is open to selection bias and other problems. ${ }^{15 \cdot 17}$ Brandt-Zawadzki and colleagues studied 
the detection of focal abnormalities in 70 patients selected retrospectively, some referred after examination by computed tomography. ${ }^{15}$ Magnetic resonance imaging showed 17 lesions not shown by computed tomography: four patients had glioma of the brain stem, but the authors did not state whether these patients' computed tomograms showed expansion of the brain stem. Three patients had an abnormality shown by computed tomography but not by magnetic resonance imaging, including one acoustic neuroma. Bradley et al reported on patients referred for evaluation of the brain and cervical spinal cord, but they implied that some patients were excluded and that some of those included had been examined by computed tomography. ${ }^{16}$ Magnetic resonance imaging was considered superior to computed tomography in 29 of about 50 patients with a lesion in the posterior cranial fossa, including one found to have a glioma of the brain stem despite a normal computed tomogram. In 20 patients (including 16 with a tumour) the two techniques were equally informative, but in one patient a computed tomogram showed a meningioma in the posterior fossa that was not shown by magnetic resonance imaging. The high incidence of abnormal cases $(73 \%$ and $69 \%$ ) in these two studies indicates that their populations were selected; in our population most patients had normal findings on magnetic resonance imaging (64\%) and computed tomography $(66 \%)$.

The third study did not support the superiority of magnetic resonance imaging. Haughton et al studied the magnetic resonance images of consecutive subjects referred when there was access to the magnetic resonance system, but they excluded 30 subjects because a verified diagnosis could not be obtained from the images and case records. ${ }^{17}$ Findings at operation or necropsy were available in 91 of the 112 cases, but the number of patients suspected of having a lesion in the posterior fossa was not specified. Overall, computed tomography was more sensitive $(91 \%)$ than magnetic resonance imaging $(82 \%)$, and the findings were not different when magnetic resonance was carried out at $0 \cdot 15 \mathrm{~T}$ and $1.5 \mathrm{~T}$.

Referral or selection bias was minimised in our study. The population was typical of patients encountered in clinical neurological and neurosurgical practice, and patients were entered into the trial before any cross sectional imaging was carried out; selection was based only on the referring doctor's suspicion of a lesion in the posterior fossa. There was a high rate of compliance, perhaps because we recognised that doctors or even patients would sometimes insist on obtaining either the new kind of scan or the more established, familiar investigation by computed tomography. Indeed, we used this to advantage in making such requests the principal end point for comparing the contributions of the two techniques to investigation and management.

When comparing two treatments the appropriate end point is their effect on outcome, whereas evaluating a diagnostic technique usually entails comparing the result of the investigation with some independent criterion. In practice few patients suspected of having a lesion in the posterior fossa undergo an operation so there is seldom a diagnosis that has been confirmed independently against which to judge the results of imaging; this greatly limits the feasibility and relevance of conventional approaches to assessment. A diagnosis based on histological findings was obtained in only 28 (3\%) of our patients ( $10 \%$ of abnormal scans).

Our design was based on the view that doctors' decisions are usually made without the benefit of a certain diagnosis. ${ }^{18}{ }^{19}$ Instead the doctor considers that the information available has made a particular disease sufficiently probable for management to be chosen. Our main comparison therefore was of the number of cases in which sufficient uncertainty remained after the first investigation for doctors to request alternative cross sectional imaging. We recognised that we were comparing not the technical performances of computed tomography and magnetic resonance imaging but doctors' attitudes to the yield of information from the two techniques. We believe that this criterion is more practically relevant in that it affects their actions; this is reflected in the influence of the second imaging investigations on doctors' plans for management. This approach to assessment is a combination of randomised trial and formal clinical audit and may be useful in evaluating other medical technologies.

The computed tomograms were reported by three experienced consultant neuroradiologists and the magnetic resonance images by a neuroradiologist, who at the beginning of the study had had five years' experience of the technique. We believe that we obtained high standards of accuracy and consistency. When an operation was carried out or necropsy performed the diagnosis by imaging was confirmed by the histological findings in each case. It was, of course, not possible to know how many of the final diagnoses were inaccurate in the other patients. Such information is extremely difficult to obtain, but there were no important changes in diagnosis or management six months after discharge.

We used a fairly early model of the magnetic resonance imager; a machine with a stronger, more stable field might have produced superior images, but this would probably not have altered substantially the yield of diagnostic information from the imaging or its influence on management. Reviewers have noted that some of the most enthusiastic reports of magnetic resonance imaging compared with computed tomography were obtained with early imagers. ${ }^{1}$ The use of gadolinium diethylenetriaminepentaacetic acid to enhance magnetic resonance images ${ }^{20}$ might have increased the preference for magnetic resonance imaging, but this agent was not generally available in Britain until January 1989.

Doctors gave a range of reasons for requesting further imaging of the posterior fossa. In most cases their aim was to exclude the possibility of a lesion, and they clearly preferred to have the results of magnetic resonance imaging before accepting such a conclusion, especially when either demyelination or a benign tumour was suspected. The requirement to provide justification for requests and to complete further forms guarded against haphazard, indiscriminate referrals. Initially the unfamiliarity of magnetic resonance images may have led doctors to request computed tomography, a more familiar technique; in accord with this the rate of requests for computed tomography after magnetic resonance imaging diminished with time. The novelty of magnetic resonance imaging did not seem to stimulate large numbers of requests for patients in whom more experience would have indicated that it was unlikely to be beneficial; indeed, requests for magnetic resonance imaging after computed tomography were more common later in the study.

We have not considered in this paper the implications of the different resources needed for computed tomography and magnetic resonance imaging or the changes in indicators of health in the two groups; these are the subject of a parallel study funded by the Medical Research Council in collaboration with the department of health economics, University of York. The initial expense of magnetic resonance imaging needs to be balanced against its freedom from the hazard of radiation and the evidence of its value in the posterior cranial fossa as well as in other areas such as the craniocervical junction ${ }^{2}$ and for selected cases in which 
a diagnosis is difficult. ${ }^{21}$ Although magnetic resonance imaging can lead to important changes in management, these occur in a small minority of patients suspected of having an intracranial lesion. The benefits of magnetic resonance imaging are therefore likely to be measured ultimately more by how it fulfils its promise of replacing invasive techniques such as myelography ${ }^{22.3}$ and diagnostic cerebral angiography ${ }^{24}$ than by its performance compared with computed tomography of the head.

We thank our colleagues in the departments of neurology, neurosurgery, neuroradiology, and clinical physics for their cooperation and collaboration and for contributing to collecting the data; Drs K W Grossart and E Teasdale for reporting many of the computed tomograms; Professor A Williams and Dr J Hutton, department of health economics, University of York, for fruitful discussions and collaboration; and $\mathrm{Dr} G$ Murray, department of medical statistics, University of Glasgow, for advice on analysis. The magnetic resonance imaging unit in the Institute of Neurological Sciences, Glasgow, was established with support from the Medical Research Council, the Scottish Hospital Endowment Research Trust, the chief scientist, the Scottish Home and Health Department, the Greater Glasgow Health Board, and the Institute of Neurological Sciences Research Trust.

1 Kent DL, Larson EB. Magnetic resonance imaging of the brain and spine -is clinical efficacy established after the first decade? Ann Intern Med 1988;108: $402-24$

2 Hadley DM, Teasdale GM. Survey of progress: magnetic resonance imaging of the brain and spine. $\mathcal{F}$ Neurol 1988;235:193-206.

3 Hillman BJ. Evaluating magnetic resonance: what do we need to know? Invest Radiol 1986;21:289-91. (Critical review.)

4 Cooper LW, Thomas MPH, Chalmers C, et al. The poor quality of early evaluations of magnetic resonance imaging. $\mathcal{F} A M A$ 1988;259:3277-80.

5 Sheps SB. Technological imperatives and paradoxes. FAMA 1988;259:3312-3.
6 Kent DL, Larson EB. Diagnostic technology assessments: problems and prospects. Ann Intern Med 1988;108:759-61.

Bydder GM, Steiner RE, Thomas DJ, et al. Nuclear magnetic resonance imaging of the posterior fossa: 50 cases. Clin Radiol 1983:34:173-88.

8 Hadley DM, Macpherson P, Grant R, Paterson J, Lawrence A. Magnetic resonance imaging in the posterior fossa. A prospective analysis of 200 patients. In: Hopf MA, ed. Proceedings of the third congress of the European Society of Magnetic Resonance in Medicine and Biology. Aberdeen: European Society of Magnetic Resonance in Medicine and Biology, 1986:94.

9 Everitt BS. The analysis of contingency tables. London: Chapman and Hall, 1977.

10 Smith T. Taming high technology. Br Med f 1984:289:393-4.

11 Institute of Medicine Committee for Evaluating Medical Technologies in Clinical Use. Assessing medical technologies. Washington, DC: National Academy Press, 1986

12 Jennett B. Assessment of clinical technologies: importance for provision and use. International fournal of Technology Assessment in Health Care 1988;4: $435-45$.

3 Jennett B. High technology medicine: benefits and burdens. 2nd ed. Oxford: Oxford University Press, 1986.

14 Ranshoff DF, Feinstein AR. Problems of spectrum and bias in evaluating the efficacy of diagnostic tests. $N$ Engl f Med 1978:299:926-30.

15 Brandt-Zawadzki M, Norman D, Newton H, et al. Magnetic resonance of the brain: the optimal screening technique. Radiologv 1984;152:71-7

16 Bradley WG, Waluch V, Yadley RA, Wycoff RR. Comparison of CT and MR in 400 patients with suspected disease of the brain and cervical spinal cord. Radiology 1984;152:695-702.

17 Haughton VM, Rimm AA, Sobocinski KA, et al. A blinded clinical comparison of MR imaging and CT in neuroradiology. Radiology 1986;160 $751-5$.

18 McCartney FJ. Diagnostic logic. Br.Med f 1987;295:1325-31

9 Balla JI, Elstein AS, Christensen C. Obstacles to acceptance of clinical decision analysis. Br.Med f 1989;298:579-82.

20 Bydder GM. Clinical application of gadolinium-DTPA. In: Stark DD, Bradley WG, eds. Magnetic resonance imaging. St Louis: C V Mosby, 1988:182-200.

21 Duncan R, Hadley DM, Teasdale GM. Magnetic resonance scanners. Lancet $1988 ; \mathrm{i}: 60-1$

22 Hyman RA, Gorey MT. Imaging strategies for MR of the spine. Rudiol Clin North Am 1988;26:505-33

23 Thomson JLG. Experiences at the new magnetic resonance imaging centre at Bristol. Br F Radiol 1989;62:134-7.

24 Dumoulin CL, Hart HR. Magnetic resonance angiography. Radiology 1986;161:717-20.

(Accepted 18 May 1989)

\title{
An unusual uveitis in Tanzanian children
}

\author{
David Yorston, Allen Foster, John Treharne
}

\begin{abstract}
In 1982-7, 254 children with panuveitis were seen at Mvumi Hospital, Tanzania, representing $56 \%$ of all cases of uveitis seen. Half were aged under 2. No consistent abnormality accounted for the uveitis and it resolved spontaneously over 6-12 weeks. A trial of prednisolone was performed in 30 children: 18 showed improvement by four weeks compared with 20 of 35 controls given only topical steroids and mydriatics.
\end{abstract}

\section{Mvumi Hospital, PO Dodoma, Tanzania \\ David Yorston, FRCS, ophthalmologist}

\section{Institute of \\ Ophthalmology, London ECIV 9EJ \\ Allen Foster, FRCS, senior lecturer in preventive ophthalmology \\ Institute of \\ Ophthalmology, London WC1 \\ John Treharne, MRCPATH, reader in virology}

Correspondence to: $\mathrm{Dr}$ Foster.

BrMed f 1989;299:355-7

\section{Introduction}

Uveitis in children is fairly unusual, representing $1-20 \%$ of all cases of uveitis. ${ }^{1-3}$ Uveitis may be divided anatomically into anterior, peripheral (pars planitis), and posterior. In analysing 150 cases in children Perkins found a ratio of anterior to peripheral to posterior uveitis of about $3: 2: 1$, with the main causes of posterior uveitis or panuveitis being toxoplasmosis (38\%) and toxocariasis (11-20\%); the cause of $24 \%$ of cases was not known.

\section{Patients and methods}

From 1982 to 1987,254 children aged 0-9 years with posterior uveitis or panuveitis were seen at Mvumi Hospital in central Tanzania. All were seen in the outpatient clinic and were admitted and examined by AF or DY. Examination included slit lamp microscopy (in older children) and direct and indirect ophthalmoscopy (sometimes performed under sedation or general anaesthesia) in all children. A tap of the anterior chamber was performed in six patients undergoing examination under anaesthesia. The aqueous humour obtained was examined for the presence and type of cells. Where indicated, investigations included a thick blood film (for malaria and tick borne relapsing fever), peripheral blood count, and examination of urine and stools by microscopy.

Serum samples from 10 children with uveitis and nine controls matched for age and sex were examined at the Institute of Ophthalmology, London. Antibodies to herpes simplex virus and Epstein-Barr virus were screened with an indirect immunofluorescence test using the virus grown in cell culture as antigen. Antibodies to HIV were screened by enzyme linked immunosorbent assay (ELISA) (Welcozyme, Wellcome), and serology for toxoplasmosis was carried out by a latex screen test.

\section{Results}

Of patients with uveitis seen from 1982 to 1987,254 were aged under $10(128(50 \%)$ aged under 2 years and $33(13 \%)$ aged 6 months or under) and 201 were aged 10 or over $(67$ aged $10-19,59$ aged $20-29,45$ aged $30-39$, 17 aged $40-49$, and 13 aged 50 or over). Of the children aged under 10 years, 124 were boys and 130 were girls.

The clinical presentation was an acute red eye or a grey-white opacity in the pupil that had been noticed by a parent, or both. The children were generally well. Ocular examination showed an acute anterior uveitis with cells, keratic precipitates, and occasionally hypo- 